\title{
Mango (Mangifera indica L.) cv. Kent fruit mesocarp de novo transcriptome assembly identifies gene families important for ripening
}

\author{
Mitzuko Dautt-Castro ${ }^{1}$, Adrian Ochoa-Leyva ${ }^{2}$, Carmen A. Contreras-Vergara ${ }^{1}$, \\ Magda A. Pacheco-Sanchez ${ }^{1}$, Sergio Casas-Flores ${ }^{3}$, Alejandro Sanchez-Flores ${ }^{4}$, \\ David N. Kuhn ${ }^{5}$ and Maria A. Islas-Osuna ${ }^{*}$
}

\footnotetext{
1 Laboratorio de Genética y Biología Molecular de Plantas, Centro de Investigación en Alimentación y Desarrollo, Hermosillo, Sonora, Mexico

2 Instituto Nacional de Medicina Genómica, Unidad de Genómica de Poblaciones, Aplicada a la Salud, Facultad de Química UNAM, Delegación Tlalpan, Mexico DF

${ }^{3}$ Laboratorio de Genómica Funcional y Comparativa, División de Biología Molecular, Instituto Potosino de Investigación Científica y Tecnológica, San Luis Potosí (SLP), Mexico

${ }^{4}$ Unidad Universitaria de Secuenciación Masiva de DNA, Instituto de Biotecnología/Universidad Nacional Autónoma de México, Cuernavaca, Morelos, Mexico

${ }^{5}$ United States Department of Agriculture - Agricultural Research Service, Subtropical Horticulture Research Station, Miami, FL, USA
}

\section{Edited by:}

Ariel Orellana, Universidad Andres Bello, Chile

\section{Reviewed by:}

Jingfa Xiao, Beijing Institute of Genomics - Chinese Academy of Sciences, China

Jiayan Wu, Beijing Institute of Genomics - Chinese Academy of Sciences, China

*Correspondence:

Maria A. Islas-Osuna, Laboratorio de Genética y Biología Molecular de Plantas, Centro de Investigación en Alimentación y Desarrollo, Carretera Ejido La Victoria Km 0.6, Hermosillo, Sonora 83304, Mexico

e-mail: islasosu@ciad.mx
Fruit ripening is a physiological and biochemical process genetically programmed to regulate fruit quality parameters like firmness, flavor, odor and color, as well as production of ethylene in climacteric fruit. In this study, a transcriptomic analysis of mango (Mangifera indica L.) mesocarp cv. "Kent" was done to identify key genes associated with fruit ripening. Using the Illumina sequencing platform, 67,682,269 clean reads were obtained and a transcriptome of $4.8 \mathrm{~Gb}$. A total of 33,142 coding sequences were predicted and after functional annotation, 25,154 protein sequences were assigned with a product according to Swiss-Prot database and 32,560 according to non-redundant database. Differential expression analysis identified 2,306 genes with significant differences in expression between mature-green and ripe mango [1,178 up-regulated and 1,128 down-regulated $(F D R \leq 0.05)$ ]. The expression of 10 genes evaluated by both qRT-PCR and RNA-seq data was highly correlated $(R=0.97)$, validating the differential expression data from RNA-seq alone. Gene Ontology enrichment analysis, showed significantly represented terms associated to fruit ripening like "cell wall," "carbohydrate catabolic process" and "starch and sucrose metabolic process" among others. Mango genes were assigned to 327 metabolic pathways according to Kyoto Encyclopedia of Genes and Genomes database, among them those involved in fruit ripening such as plant hormone signal transduction, starch and sucrose metabolism, galactose metabolism, terpenoid backbone, and carotenoid biosynthesis. This study provides a mango transcriptome that will be very helpful to identify genes for expression studies in early and late flowering mangos during fruit ripening.

Keywords: Mangifera indica L., mesocarp, fruit ripening, transcriptome, cell wall hydrolytic enzymes, ethylene, fruit quality

\section{INTRODUCTION}

Fruit ripening is a complex biochemical and physiological process where modifications in cell wall and secondary metabolism lead to changes in macroscopic parameters such as appearance, texture, flavor, and aroma. The molecular basis of fruit ripening has been widely studied by traditional biochemistry, genetics and molecular biology in fruits like tomato, papaya, strawberry, apple, peach, among others (Gapper et al., 2013). Mango (Mangifera indica L.), a member of the Anacardiaceae family, is the second most important tropical fruit crop in the horticulture industry worldwide (Singh et al., 2013). So far, gene expression changes in mango mesocarp have been studied only for specific genes to provide hints into the ripening process. RNA sequencing (RNA-seq) is a useful technology to measure global changes in transcription (Marguerat and
Bähler, 2010). RNA-seq has been used to understand the ripening process in fruits like Chinese bayberry, watermelon and orange (Guo et al., 2011; Feng et al., 2012; Yu et al., 2012). Therefore, the RNA-seq of mango mesocarp can provide insights about specific gene expression patterns for mature-green and ripe mango.

Mango is a climacteric fruit, and the expression of ethylene biosynthesis genes like ACC synthase (ACS) and ACC oxidase (ACO) are predicted to change during ripening, as previously shown in tomato. Studies in tomato show that LeACS2 and LeACO1 dominate gene expression during climacteric ethylene production. Other important ethylene-related genes are membrane receptors involved in regulation of secondary metabolites (White, 2002). The Arabidopsis ethylene receptor family is comprised of five members divided into two subfamilies: ETR1 and 
ERS1, subfamily I; ETR2, ERS2, and EIN4, subfamily II (Bleecker, 1999). However, it is necessary to identify those genes and pathways during mango mesocarp ripening.

Firmness is a key post-harvest quality attribute, and it dictates commercialization strategies, since it must reach the consumer in 2 weeks at the most. Firmness loss is due to the action of cell wall hydrolytic enzymes such as polygalacturonases (PGs), pectin methyl esterases (PMEs), pectate lyases (PLs), $\alpha$-galactosidase ( $\alpha$ GAL), $\beta$-galactosidases ( $\beta$-GAL), glucosidases (Glu), among others (Goulao and Oliveira, 2008). There is also a key physical process that is involved in fruit softening and is due to the action of expansins, which are cell wall proteins that loosen cellulose structure without any hydrolytic activity (McQueen-Mason and Cosgrove, 1995).

Color changes during fruit ripening include the conversion of chloroplasts to chromoplasts. As a result of the loss of photosynthetic capacity of the chloroplasts, thylakoid structures become sites for the accumulation of carotenoids in the fruit cells (Klee and Giovannoni, 2011). The pigment accumulation in mango fruit is cultivar-dependent, but in general, mango has a high content of carotenoids in mesocarp tissue responsible for the intense yellow color (Singh et al., 2013). The enzymes involved in carotenogenesis change during fruit ripening. For example, phytoene synthase (PSY) and carotenoid betahydroxylase-1 (CHYB1) accumulate at the tomato breaker stage compared to the red-ripe stage, leading to high levels of lycopene (Smita et al., 2013). The fruit flavor is not always directly related to their sugar content. Volatile compounds like monoterpenes, sesquiterpenes, terpenoids, carotenoids, and amino acids are also critical for ripe-mango flavor (El Hadi et al., 2013). The terpene hydrocarbons are important factors for mango flavor in cultivars like "Kent," "Keitt," and “Tommy Atkins" (Singh et al., 2013). Terpene synthases that convert prenyl diphosphates to terpenes were identified by RNA-seq in Solanum lycopersicum stem trichomes and were expressed in several tissues and enriched in some others (Bleeker et al., 2011).

In order to understand the mango ripening process, it is very important to know the gene families associated with the quality parameters mentioned above as well as their expression patterns. Therefore, the objective of this study was to obtain the transcriptome from mature-green and ripe mango mesocarp in order to identify differentially expressed transcripts involved in mango ripening for ethylene production, softening, sugar synthesis, volatile compounds, and color pigments to elucidate and to investigate in depth the ripening process in this fruit.

\section{MATERIALS AND METHODS \\ PLANT MATERIAL AND RNA PREPARATION}

Mango (M. indica L.) fruit cultivar "Kent" was harvested at the National Institute of Forestry, Agricultural and Veterinary Research (INIFAP) research station located in Navojoa, Sonora, Mexico $\left(27^{\circ} 03^{\prime} 49.33^{\prime \prime} \mathrm{N}\right.$ and $\left.109^{\circ} 30^{\prime} 11.42^{\prime \prime} \mathrm{W}\right)$. This is a commercial mango variety, is not an endangered species and no permit is required for sampling. Samples were homogeneous in shape, size and physiological maturity and transported to the laboratory. Fruits were disinfected with chlorinated water and stored at $20^{\circ} \mathrm{C}$ up to 10 days.
Total RNA was isolated from mango mesocarp tissue pulverized with liquid-nitrogen. Sampling was done at day 1 (maturegreen mango) and day 10 (ripe mango), using two individual fruits for each ripening stage. RNA was isolated as previously described Lopez-Gomez and Gómez-Lim (1992), and then it was treated with RNase-free DNase I (Roche) to eliminate contaminant genomic DNA. The RNA quantity was estimated using a NanoDrop ND-1000 UV-Vis spectrophotometer at $260 \mathrm{~nm}$ (Nano Drop Technologies Inc., Wilmington, DE, USA). RNA integrity was analyzed by $1 \%$ agarose gel electrophoresis under denaturing conditions and with the 2100 Bioanalyzer system (Agilent Technologies, Santa Clara, CA, USA).

\section{RNA-seq LIBRARY PREPARATION, SEQUENCING, DE NOVO ASSEMBLY, AND DIFFERENTIAL EXPRESSION ANALYSIS}

Two mature-green and two ripe mango were selected to prepare four independent cDNA libraries using the Illumina TruSeq RNA sample preparation Kit v2 following the manufacturer instructions. These four libraries were sequenced using the Genome Analyzer GAIIx II (Illumina) at the Institute of BiotechnologyUNAM (Cuernavaca, Morelos, Mexico) with a configuration for pair end reads with a 72 bp read length.

The mango mesocarp transcriptome was assembled with the Trinity software (Grabherr et al., 2011). A pooling strategy was used only for the transcriptome de novo assembly in order to increase the chances of reconstructing low expressed transcripts. Each set of reads from the two biological replicates of mature-green and ripe mango were mapped back to those transcripts using the Bowtie aligner with some Perl script provided with the Trinity pipeline.

To calculate the differential expressed (DE) genes between the mature-green and ripe mango the transcripts from each library were normalized using the RPKM method.

The initial reads for each replicate and each condition were mapped back to the transcriptome assembled by using the "align_and_estimate_abundance.pl" Perl script from the Trinity pipeline. Each condition with its replicates were mapped and counted separately as described previously. Using the RSEM counts for each sample, a merged table was generated using the "abundance_estimates_to_matrix.pl" Perl script that is also included in the Trinity pipeline. For the differential expression analysis we used the "run_DE_analysis.pl" script with the merged table. The "run_DE_analysis.pl” script takes a read count cut-off of 10 reads after adding the values of all replicates and conditions (min_rowSum_counts 10 [default]). The DE genes were filtered using a False Discovery Rate (FDR) cut-off line $p \leq 0.05$.

\section{TRANSCRIPT ANNOTATION AND PATHWAY ANALYSIS}

To identify homologous proteins, all mango "Kent" mesocarp deduced amino acid sequences were BLASTed against the nonredundant (NR) and Swiss-Prot database using the Blast2GO software (Conesa and Götz, 2008). The E-value cut-off was set at $1.0 \mathrm{E}^{-3}$. Mango proteins were functionally mapped to GO terms and annotated with the following parameters: E-Value-Hit-Filter: $1.0 \mathrm{E}^{-3}$. Annotation cut-off: 55; GO weight: 5; Hsp-Hit Coverage cut-off: 0 . Statistically enriched GO terms represented in the DE genes were also identified using Blast2GO setting with the term 
filter value to $p \leq 0.05$ and the term filter mode FDR. Mango mesocarp proteins were associated with protein families and domains with InterProScan. Additionally, the annotation of Clusters of Orthologous Groups of proteins (COG) was obtained (Tatusov et al., 2000).

The transcripts were analyzed with the Kyoto Encyclopedia of Genes and Genomes (KEGG) pathway database (Kanehisa and Goto, 2000). The KEGG Automatic Annotation Server (KAAS, http://www.genome.jp/kaas-bin/kaas_main?mode = partial) was employed to map KEGG pathways, using the $\mathrm{BBH}$ (bi-directional best hit) method to assign orthologs. The percentages of coverage were predicted by contrast to the KEGG pathways of Citrus sinensis database.

\section{VALIDATION OF DIFFERENTIAL GENE EXPRESSION BY REAL-TIME TRANSCRIPTION PCR (qRT-PCR) ANALYSIS}

First-strand cDNA was synthesized from $5 \mu \mathrm{g}$ of DNA-free RNA (from the same mature-green and ripe mango fruits that were used for RNA-seq) using Super Script III reverse transcriptase (Invitrogen) according to the manufacturer's protocol. The cDNA was adjusted to a $100 \mathrm{ng} / \mu \mathrm{l}$ concentration and it was diluted in an 1:5 ratio, to evaluate the primers of the target genes and the reference gene glyceraldehyde-3-phosphate dehydrogenase (GAPDH). qPCR was done using iTaq Universal SYBR Green Supermix (BIO-RAD).

The expression of 10 genes that were DE during mango ripening according to the transcriptome data were PCR-amplified in triplicate in reactions that included $5 \mu$ l of cDNA template (4$20 \mathrm{ng}$ ), $10 \mu \mathrm{L}$ of iTaq Universal SYBRGreen Supermix, $1 \mu \mathrm{L}$ of $5 \mu \mathrm{M}$ of each sense and antisense primer and RNase-free water to a $20 \mu \mathrm{L}$ final volume. Specific primers are listed in Table S1. The PCR was done in a Step-One ${ }^{\mathrm{TM}}$ Real-time PCR System (Applied Biosystems Inc, Foster City, CA, USA). Amplification conditions were $95^{\circ} \mathrm{C}$ for $10 \mathrm{~min}$ and 40 cycles of $95^{\circ} \mathrm{C}$ for $15 \mathrm{~s}$ and $60^{\circ} \mathrm{C}$ for $1 \mathrm{~min}$. PCR specificity was confirmed by constructing a melt curve after amplification in a range from $95^{\circ} \mathrm{C}$ for $15 \mathrm{~s}, 60^{\circ} \mathrm{C}$ for $1 \mathrm{~min}$ and $95^{\circ} \mathrm{C}$ for $15 \mathrm{~s}$. Non-template controls were also included. The results were normalized to the $G A P D H$ expression levels and analyzed with the $2^{-\Delta \Delta C T}$ method (Livak and Schmittgen, 2001).

Statistical analysis was performed using one-way ANOVA, with a significance level of $p<0.05$, using the NCSS (2007) software. A linear regression analysis was done to obtain the correlation between transcript abundance assayed by qRT-PCR and the transcription profile revealed by RNA-seq data. The correlation coefficient $(R)$ was obtained by non-parametric analysis (Spearman).

\section{RESULTS AND DISCUSSION MANGO RNA-seq AND DE NOVO ASSEMBLY}

A total of 234,310,610 Illumina reads from both, mature-green and ripe conditions were obtained with a total of $16,870,363,920$ bases (16 Gb). The FastQC program was used to evaluate the quality of the reads and low-quality or adapter sequences were removed. After trimming and discarding low-quality reads, 67,682,269 clean reads $(4,873,123,368 \mathrm{pb})$ were used. The GenBank accession number for those reads is SRP045880. After Trinity assembly 80,969 transcripts were obtained with a mean length of 836 bp and $\mathrm{N}_{50}$ of 1,456 bp. The largest transcript was 8,713 nucleotides long. Transcripts were assembled into 52,948 putative unigenes of which 33,142 had an open reading frame (ORF). Detailed information about the assembly statistics are presented in Table 1. A leaf transcriptome of mango "Langra" was reported (Azim et al., 2014) where 85,651 transcripts were obtained with a mean size of $536 \mathrm{bp}$ resulting in 30,509 unigenes. In this study, we obtained 22,439 more unigenes and a significantly higher mean transcript length. The pooled transcriptomes from mango "Zill" pericarp and pulp had 124,002 transcripts with a mean size of 838 bp resulting in 54,207 unigenes (Wu et al., 2014a) essentially similar to our results.

\section{ANNOTATION AND FUNCTIONAL CHARACTERIZATION}

The annotation of assembled sequences was performed using the Swiss-Prot and NCBI non-redundant databases and the results are shown in Figure 1. According to the Swiss-Prot database, 25,154 genes were fully annotated, 3,905 were partially annotated (Psignal, Pfam, COG, among others) and for 5,264 there was no annotation. In contrast, the results of the annotation performed with the NR database suggest that 32,560 deduced proteins (98.2\%) showed significant BLASTp matches with amino acid sequences deposited in the nr database (cut-off E-value of $10^{-3}$ ). Citrus protein sequences were the most commonly matched with $36.9 \%$ $(12,249$ sequences) of the 33,142 proteins matched with $C$. sinensis followed by $35.3 \%$ with Citrus clementina (11,708 amino acid sequences; Figure 2). A phylogenetic study of mango chloroplast DNA (cpDNA) reported that $C$. sinensis was closely related to M. indica (Azim et al., 2014). M. indica L., meanwhile only presented hits for 62 amino acid sequences, as little publicly available sequence information previously existed for this species.

The predicted mango genes were classified into the three Gene Ontology (GO) categories: Cellular Component, Biological Process, and Molecular Function. Based on sequence homology, 29,844 mango unigenes (Figure 1) were categorized into 81,315 functional groups. The Cellular Component categorycontained 10,999 unigenes with the most highly represented terms being "cell part" (9,002), "membrane-bounded organelle" $(5,332)$ and "protein complex" (2,448; Figure S1). The Biological Process category contained 16,055 unigenes with the more highly represented GO terms including "organic substance metabolic

Table 1 | Summary of mango mesocarp RNA-seq and de novo assembly.

\begin{tabular}{ll}
\hline Sequence & Value \\
\hline Total bases & $4,873,123,368$ \\
Total RNA-seq reads & $67,682,269$ \\
Total transcripts & 80,969 \\
Average length of transcripts & 835.90 \\
Largest transcript & 8713 \\
N50 length of transcripts & $1456, n=14912$ \\
Putative unigenes & 52,948 \\
Transcripts with a ORF & 33,142
\end{tabular}




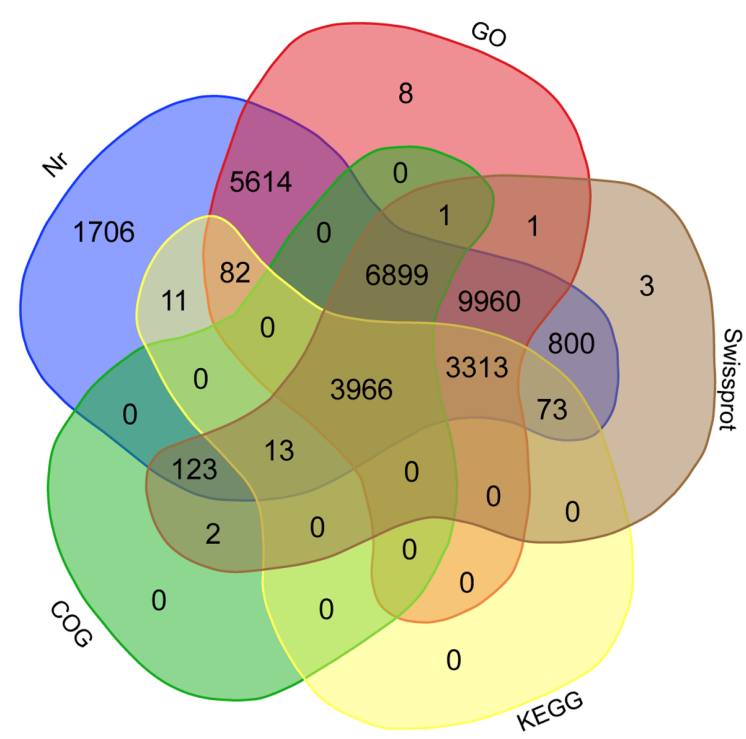

FIGURE 1 | Venn diagram of mango shared unigenes. Annotation was done according to the NCBI non-redundant (Nr), Swiss-Prot, COG, and KEGG databases and classified into Gene Ontology (GO). Overlapped unigenes are indicated in the intersections.

process" (10,261), "primary metabolic process" $(9,805)$ and “cellular metabolic process" (9,557; Figure S2). The Molecular Function category contained 21,331 unigenes with the most frequent GO terms being "organic cyclic compound binding" $(7,686)$, "heterocyclic compound binding" $(7,677)$, and "ion binding" $(6,890$; Figure S3). In the mango leaf transcriptome (Azim et al., 2014), similar GO terms at similar frequencies to the results of this work were observed, where the terms "cell part" for cellular component, "cellular process" for biological process and "binding" for molecular function, were their most representative GO terms.

According to the COG database 11,004 proteins showed a functional annotation. The most representatives COG annotations were Serine/threonine protein kinase (COG0515) with 1,318 proteins. These kinases catalyze the phosphorylation of serine or threonine residues on target proteins by using ATP as phosphate donor, which is a way to regulate the function of the target protein (UniProt Consortium, 2014). Followed by FOG: WD40 repeat (COG2319) with 301 proteins, which are implicated in a variety of functions that include signal transduction, regulation of transcription and cell cycle control, among others (Stirnimann et al., 2010). And by leucine-rich repeat (LRR) protein (COG4886) with 190 proteins, which are proteins whose main function appears to be to provide a versatile structural framework for the formation of protein-protein interactions (Kobe and Kajava, 2001).

Transcripts were also analyzed in the KEGG database and a total of 7,458 unigenes (Figure 1) were assigned to 327 KEGG pathways. Tables S2 and S3 show 30 metabolic pathways of genes for both the total mango transcriptome and the differentially expressed subset. The most significant pathways with regard to the number of hits from the whole transcriptome data were "biosynthesis of amino acids," "ribosome" and "RNA transport," different to the results of the mango "Zill" transcriptome, who reported that "metabolic pathways," "biosynthesis of secondary metabolites" and "plant-pathogen interactions" were the maps with the highest transcripts representation (Wu et al., 2014a). In the other hand, 3,966 unigenes were annotated in all the databases, including NR, Swiss-Prot, GO, COG and KEGG (Figure 1).

Fruit ripening is a complex process that involves physiological and biochemical modifications associated with metabolic pathways, like the conversion of starch to sugars, changes in the biosynthesis and accumulation of pigments, biosynthesis of flavor and aromatic volatiles and remodeling of the structure of cell wall (Goulao and Oliveira, 2008). Table 2 shows five major pathways associated with fruit ripening, the number of gene products involved in each and the percentage of gene products of these pathways identified from the transcriptome or the differentially expressed subset with respect to the $C$. sinensis database. The metabolic pathways are: plant hormone signal transduction (Figure S4), starch and sucrose metabolism (Figure S5), galactose metabolism (Figure S6), terpenoid backbone biosynthesis (Figure S7) and carotenoid biosynthesis (Figure S8).

\section{DIFFERENTIAL GENE EXPRESSION DURING MANGO RIPENING}

Using the edgeR pipeline included with the Trinity software, a total of 2,306 genes were identified as differentially expressed between the mature-green and ripe mango stages, representing $6.9 \%$ of total unigenes with a predicted ORF (FDR $<0.05)$. From these, 1,178 were up-regulated and 1,128 were down-regulated (Figure S9).

As was expected in a climacteric fruit, there was a wide range of genes up-regulated during mango ripening, such as carbohydrate catabolism, sucrose and ethylene biosynthesis among others. Genes encoding 1-aminocyclopropane-1-carboxylate (ACC) oxidase, PME, PG, PL, endoglucanase, expansin, $\beta$-galactosidase, $\alpha$ -galactosidase, rhamnogalacturonate lyase (RGL), and $\beta$-amylase among others were expressed at higher levels at ripe mango and are listed in Table 3. These genes are generally encoded in multiple copies in plants. Family members of PGs, PLs, $\beta$-galactosidases and other unigenes identified in this transcriptome will be very helpful in future studies where their expression profiles at different developmental stages will be assayed at the transcriptional and translational level.

We determined if particular GO terms were enriched in the DE genes as compared to the complete transcriptome. Figure 3 shows the significantly enriched terms ranked according to the $p$-value and number of genes. Some enrichment of GO terms of up-regulated genes (Figure 3A) are associated to fruit ripening like "cell wall" and "integral component of endoplasmic reticulum membrane," "starch metabolic process" and "sucrose metabolism." Similar up-regulation of genes associated with "cell wall" and the "starch metabolic process" during fruit ripening has been previously observed (Feng etal., 2012; Wu et al., 2014b). In addition, genes involved in other important biological processes such as "regulation of gene expression," "carbohydrate catabolic process" and "DNA binding" were also identified. Some enrichment of GO terms for down-regulated genes 


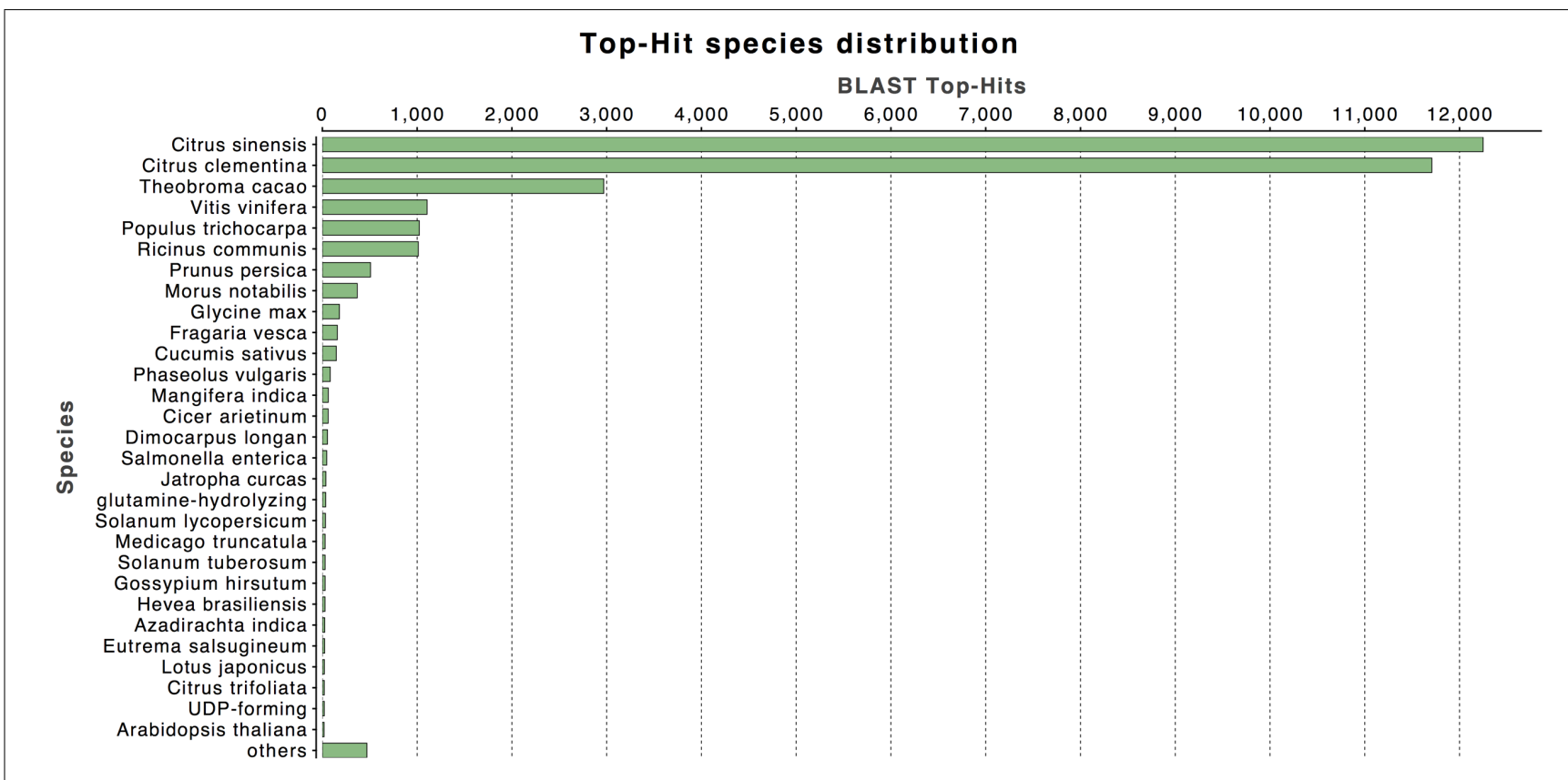

FIGURE 2 | Top-hit species taxonomic distribution. Number of Unigenes matching the 30 top species using BLASTx in the NR database.

Table 2 | Some important metabolic pathways involved in mango fruit ripening according to the Kyoto Encyclopedia of Genes and Genomes (KEGG) Citrus sinensis database.

\begin{tabular}{lll} 
KEGG pathway & $\begin{array}{l}\text { Number of genes in total } \\
\text { transcriptome/percentage } \\
\text { of coverage (\%) }\end{array}$ & $\begin{array}{l}\text { Number of DE } \\
\text { genes/percentage } \\
\text { of coverage (\%) }\end{array}$ \\
\hline $\begin{array}{l}\text { Plant hormone signal } \\
\text { transduction }\end{array}$ & $36(85.70)$ & $20(47.61)$ \\
$\begin{array}{l}\text { Starch and sucrose } \\
\text { metabolism }\end{array}$ & $37(100)$ & $14(49.99)$ \\
$\begin{array}{l}\text { Galactose metabolism } \\
\text { Terpenoid backbone }\end{array}$ & $30(100)$ & $6(55.99)$ \\
biosynthesis & & $7(22.58)$ \\
$\begin{array}{l}\text { Carotenoid } \\
\text { biosynthesis }\end{array}$ & $15(91.11)$ & $6(51.11)$ \\
\end{tabular}

(Figure 3B) was associated to, "protein phosphorylation," "regulation of transcription" and "protein serine/threonine kinase activity."

\section{Ethylene biosynthesis and signaling pathway genes}

Four genes encoding the ethylene biosynthesis enzyme (ACC) oxidase and two encoding ACC synthase were obtained in this study. Three ACC oxidase genes were up-regulated in ripe mango (comp35681, comp36021, comp59876) with 2, 4, and 7-fold changes, respectively. The expression of the two ACC synthase genes did not change. Both ACC oxidase and ACC synthase are encoded by a multigene family of five and nine members, respectively, in tomato (Nath et al., 2006).
Ethylene signaling is a plant hormone signal transduction pathway. Several ethylene response genes have been identified in Arabidopsis (Bleecker, 1998; Schaller and Kieber, 2002) and tomato (Lashbrook et al., 1998; Tieman and Klee, 1999). In this work we have identified four ethylene receptor transcripts: ETR1 (comp46286) and ETR2 (comp47044), that remain constant during fruit ripening, and ERS1 (comp32379) and EIN4 (comp34039) that showed a decrease in their expression levels.

Other components of the ethylene signaling pathway, such as the constitutive triple response CTR1 (comp43157), which was down-regulated (-2-fold) and eleven ethylene-responsive transcription factor (ERF) were found in this transcriptome, where one (comp27110) was up-regulated (7-fold), seven were downregulated (comp18706, comp26333, comp34532, comp39082, comp40325, comp42703, and comp45406) in mango ripe and three did not changed (comp24020, comp41521, comp45323). In a previous report (Wu et al., 2014b), one ERF showed a decrease in its expression levels during orange ripening.

Regulator proteins EIN2 (comp43660), EIN3 (comp47416), and EIL3 (comp41806) were also identified and their levels remained constant. EIN3 proteins are controlled by two F-box proteins (EBF1/2), which are SKP-Cullin-F-box (SCF) E3 ligases targeting EIN3 and EIL for 26S proteosome degradation (Guo and Ecker, 2003). Two EBF (ethylene binding F-Box proteins) sequences were found in this study, EBF1 (comp45329) was upregulated (2-fold) and EBF2 (comp44836) was down-regulated (-2-fold) in ripe mango.

\section{Genes associated with firmness}

Mango fruit ripening involves changes in cell wall structure and degradation of lamella media, which are mainly composed by pectin, as well as loss of starch, which result in loss of firmness 
Table 3 | List of some up-regulated genes involved in major processes associated with fruit ripening according to RNA-seq data (FDR $\leq 0.05$ ).

\begin{tabular}{|c|c|c|c|c|}
\hline Gene identifier & Function & Gene description & Fold-change $\left(\log _{2}\right)$ & FDR \\
\hline comp59876 & Ethylene biosynthesis and signaling & $\begin{array}{l}\text { 1-aminocyclopropane-1-carboxylate } \\
\text { oxidase (ACO) }\end{array}$ & 7 & $6.72 \mathrm{E}-09$ \\
\hline comp35681 & Ethylene biosynthesis and signaling & $\begin{array}{l}\text { 1-aminocyclopropane-1-carboxylate } \\
\text { oxidase (ACO) }\end{array}$ & 2 & 0.0032 \\
\hline comp36021 & Ethylene biosynthesis and signaling & $\begin{array}{l}\text { 1-aminocyclopropane-1-carboxylate } \\
\text { oxidase (ACO) }\end{array}$ & 4 & 1.30E-11 \\
\hline comp27110 & Ethylene biosynthesis and signaling & $\begin{array}{l}\text { Ethylene-responsive transcription factor } \\
\text { (ERF) }\end{array}$ & 7 & 1.45E-11 \\
\hline comp45079 & Cell wall metabolism & Pectin methyl esterase (PME) & 8 & $1.75 \mathrm{E}-12$ \\
\hline comp28658 & Cell wall metabolism & Polygalacturonase (PG) & 12 & 3.00E-06 \\
\hline comp63384 & Cell wall metabolism & Pectate lyase (PL) & 11 & 4.42E-13 \\
\hline comp28245 & Cell wall metabolism & Pectate lyase (PL) & 6 & 8.37E-07 \\
\hline comp49359 & Cell wall metabolism & Pectate lyase (PL) & 5 & 7.62E-12 \\
\hline comp21883 & Cell wall metabolism & Pectate lyase $(\mathrm{PL})$ & 2 & $4.42 \mathrm{E}-13$ \\
\hline comp41701 & Cell wall metabolism & Beta-galactosidase ( $\beta$-GAL) & 12 & 0.0002 \\
\hline comp41168 & Cell wall metabolism & Beta-galactosidase ( $\beta$-GAL) & 10 & 0.0001 \\
\hline comp35981 & Cell wall metabolism & Beta-galactosidase ( $\beta$-GAL) & 9 & 0.0001 \\
\hline comp33387 & Cell wall metabolism & Endoglucanase (GUN) & 9 & 0.0001 \\
\hline comp17443 & Cell wall metabolism & Endoglucanase (GUN) & 10 & 0.0002 \\
\hline comp48152 & Isoprenoid biosynthetic process & $\begin{array}{l}\text { Farnesyl pyrophosphate synthase } \\
\text { (FPPS) }\end{array}$ & 2 & 0.0001 \\
\hline comp48107 & Isoprenoid biosynthetic process & $\begin{array}{l}\text { Geranylgeranyl pyrophosphate } \\
\text { synthase (GGPS) }\end{array}$ & 8 & $2.59 \mathrm{E}-16$ \\
\hline comp45134 & Sucrose metabolic process & Sucrose synthase 7 (SuSy) & 7 & $1.72 \mathrm{E}-16$ \\
\hline comp42184 & Carotenoid metabolic process & Lycopene isomerase (CRTISO) & 4 & 0.0014 \\
\hline comp45152 & Carotenoid metabolic process & Beta-carotene 3-hydroxylase (CHYB) & 4 & 0.0061 \\
\hline comp44532 & Polysaccharide catabolic process & Beta-amylase (BAM) & 3 & 1.02E-06 \\
\hline comp39992 & Polysaccharide catabolic process & Beta-amylase (BAM) & 2 & 0.0001 \\
\hline
\end{tabular}

(Yashoda et al., 2006; Cárdenas-Coronel et al., 2012). Gene families encoding for PGs, PMEs, PLs, RGL, $\alpha$-GAL, $\beta$-GAL and $\beta$-glucosidases ( $\beta$-Glu) that degrade cell wall components, as well as $\alpha$ and $\beta$-amylases that degrade starch were $\mathrm{DE}$ in this work $($ FDR $<0.05)$.

In this study, nine $P G$ unigenes were obtained and six of them (comp28658, comp34301, comp35446, comp39571, comp39826, comp39773) were up-regulated in ripe mango, with 12, 10, $10,9,2$, and 2-fold changes, respectively. One $P G$ was downregulated (comp26280) with a -4 fold. These results suggest that PG expression is developmentally regulated and that most of the PGs act in late phase of ripening but they could act also at the beginning. In papaya (Carica papaya), Fabi et al. (2014), reported that $c p P G 1$ plays a central role during pulp softening. This $c p P G 1$ 
A

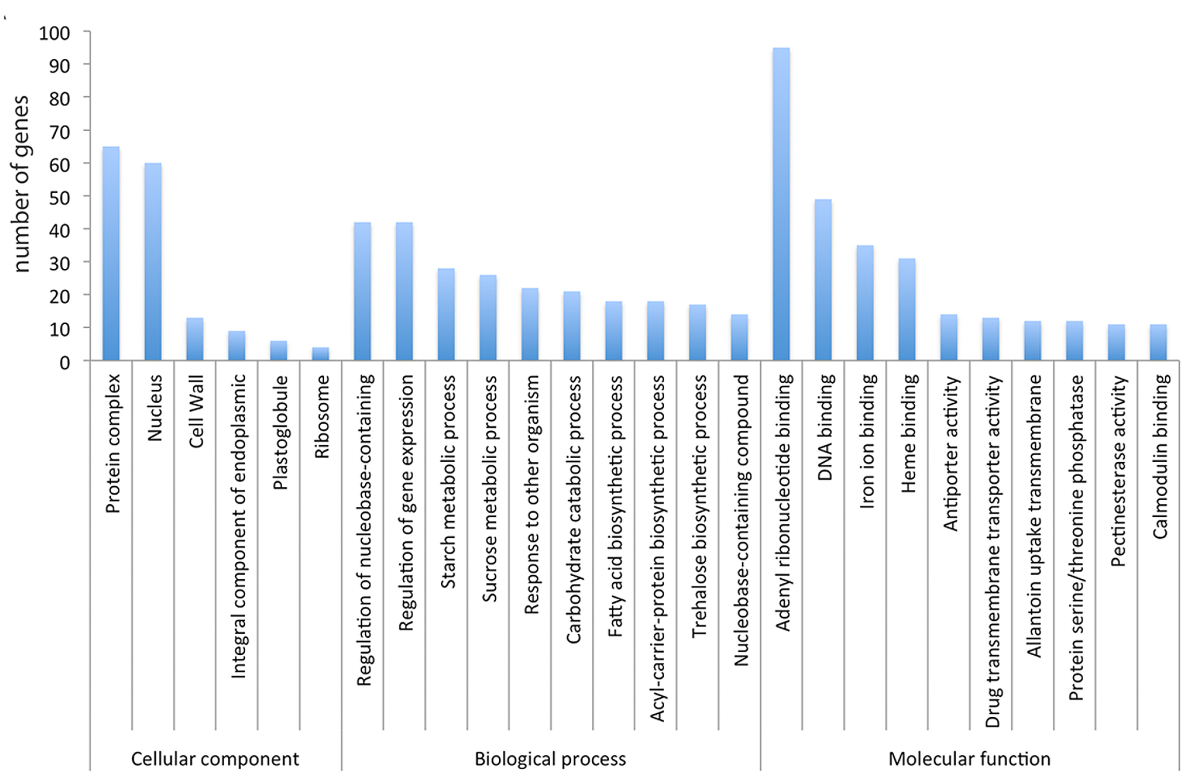

B

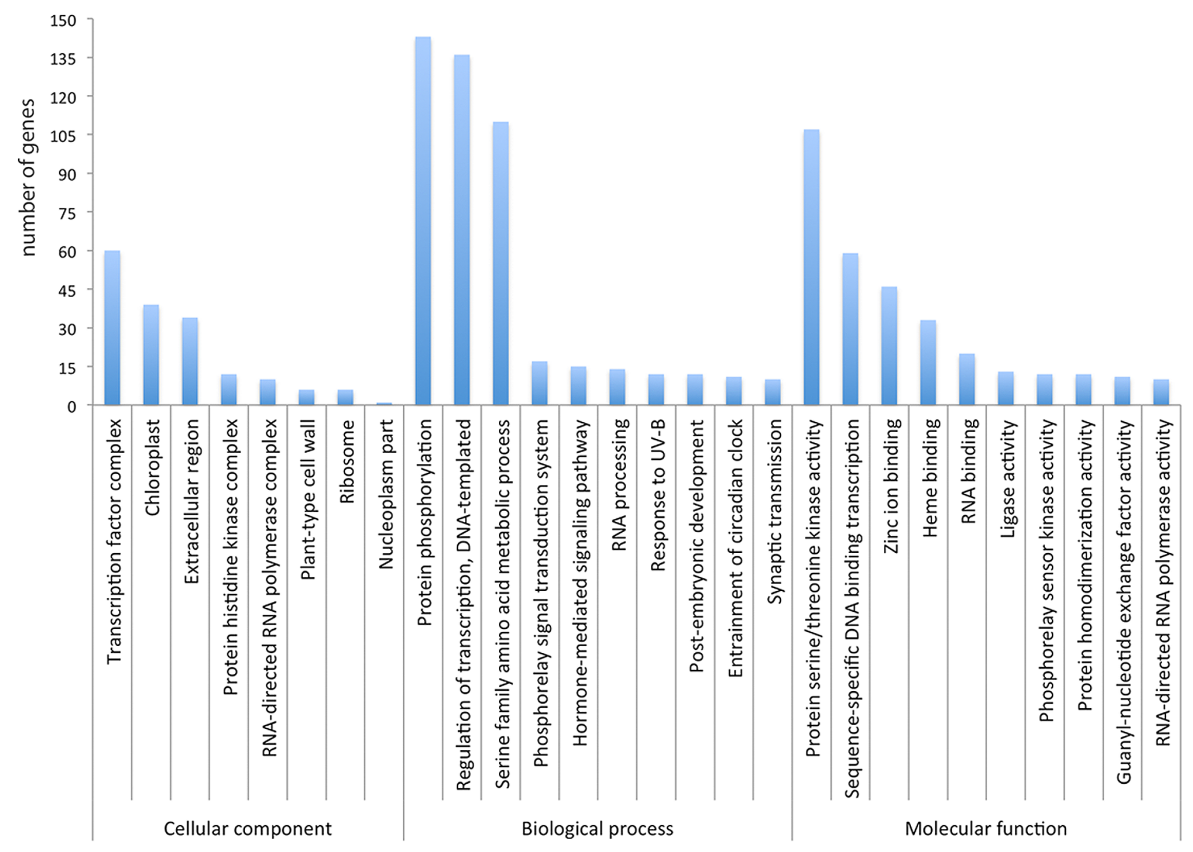

FIGURE 3 | (A) Significantly enriched GO terms in the up-regulated transcripts ranked according to the $p$-value and number of genes. (B) Significantly enriched GO terms in the down-regulated transcripts ranked according to the $p$-value and number of genes.

presented higher expression levels compared with other three papaya $P G$. Comparing the amino acid sequences of cpPG1 against mango PG, those encoded in comp35446 and comp28658, were 68 and $67 \%$ identical, respectively. The up-regulation of these two PG (comp35446 and comp28658) in ripe mango could imply their role in pulp softening. In banana (Musa acuminata), four genes encoding PG associated with fruit ripening were also reported (Mehar and Pravendra, 2005). Comparison of amino acid sequences of these PGs and mango PGs showed a high percentage of identity (up to $62 \%$ ) and their expression patterns were also similar. In mango cultivars "Alphonso" and "Dashehari," three PG isoforms that showed an increase in their activity during fruit ripening were reported (Prasanna et al., 2006; Singh and Dwivedi, 2008).

Pectin, formed by galacturonic acid with $\alpha-1,4$ linkages, generally is highly methyl-esterified and must be de-esterified by PME to be later processed by PG (Brummell and Harpster, 
2001). Seven PME cDNAs were found in this transcriptome, six of them (comp45079, comp198908, comp27472, comp173846, comp37143, comp37279) were up-regulated with 8, 7, 6, 5, 3, and 2 -fold changes, respectively and one (comp43676) was downregulated (-2-fold) in ripe mango. These results suggest that PME expression is differentially regulated during mango ripening.

The abundance of four PL (comp63384, comp28245, comp49359, comp21883) transcripts increased during mango ripening with $11,6,5$, and 2 -fold changes, respectively. In a previous study with mango "Dashehari," a PL gene associated to fruit ripening was reported and its expression levels increased in ripe mango (Chourasia et al., 2006). This PL gene is similar to the PL gene (comp21883) reported in this transcriptome (both are $99 \%$ identical in their amino acid sequences). In banana, two different $P L$ (Pel I and Pel II) cDNAs associated with fruit ripening have been reported. Both PL presented higher levels of expression in ripe bananas (Marin-Rodriguez et al., 2003).

In this study, only one $R G L$ (comp25112) was obtained and it was DE in ripe mango (5-fold). It is known that RGL degrades pectin type rhamnogalacturonan I, but little is known about how it acts in fruit cell walls. In potato (Solanum tuberosum L.) plants transformed with fungal $R G L$, the resulting tubers had cells with an altered morphology and their cell wall contents were easier to extract than those from wild-type plants, suggesting the importance of this enzyme (Oomen et al., 2002). Recently, in strawberry (Fragaria x ananassa) an RGL gene was reported to be up-regulated in receptacle in ripe fruit and it was related to the degree of firmness of fruit according to genetic linkage association analysis (MolinaHidalgo et al., 2013). This strawberry RGL enzyme is 57\% identical in amino acid sequence to the mango RGL (comp25112) from this transcriptome. This enzyme could be important in softening and it will be interesting to uncover its function.

Two of the main pectin deglycosylating enzymes that participate in this process are $\alpha$-GAL and $\beta$-GAL (Goulao and Oliveira, 2008). In this study, four $\alpha-G A L$ transcripts were identified and three of them (comp38455, comp42509, comp45738) did not changed expression levels during ripening; while one of them (comp46653) was up-regulated (2-fold change). Nine $\beta$ GAL genes were identified and three (comp41701, comp41168, comp46653) were up-regulated with fold-changes of 12, 10, and 2, respectively, in ripe mango; while five $\beta-G A L$ transcripts (comp42509, comp250695, comp44177, comp45738, comp44177) expression levels did not changed in ripe mango. Among the glycosidases from mango, $\beta$-GAL appeared to be the most predominant (Ali et al., 1995; Prasanna et al., 2005). This fact is consistent with the number of unigenes for $\beta$-GAL obtained in this study.

Two $\beta$-Glu transcripts (comp43956, comp47526), which are involved in cellulose degradation, were up-regulated in ripe mango with 6 and 5-fold change, respectively. Expression analysis of $\beta$ Glu during ripening in fruits is very limited; most of the reports are about enzymatic analysis. In mango "Ataulfo," the activity of this enzyme increased significantly during fruit ripening, which coincides with a dramatic decrease in firmness (Muy-Rangel et al., 2009). In sweet cherry (Prunus avium L.), the purification of one $\beta$-Glu has been reported and its activity increased during ripening (Gerardi et al., 2001).
In this work, an increase in the expression levels of five EXP transcripts (comp32472, comp51667, comp31446, comp32692, comp48981) was observed with 17, 5, 4, 2, and 2-fold changes, respectively. Only one mango EXP (comp42385) showed decreased expression (-2-fold change) in ripe fruit. In mango "Dashehari" and "Zill," expansin genes had increased expression during fruit ripening (Sane et al., 2005; Zheng et al., 2012). EXPA1 of mango "Dashehari" is $71 \%$ identical to EXP (comp51667) obtained in this study that also had increased expression levels in ripe fruit.

In mango, there is evidence that enzymatic hydrolysis of starch by $\alpha$ or $\beta$ - amylases results in major loosening of the cell wall structure, which results in a decrease of the fruit firmness and increase of sweetness. Also, in mango "Kent" there is evidence that $90 \%$ of starch is catabolized in the early stages of fruit ripening, which is associated with fruit softening (Yashoda et al., 2006; CárdenasCoronel et al., 2012). In this work, four $\beta$-amylases encoding genes were found, two of them (comp44532, comp39992) were up-regulated (3, 2-fold changes), whereas the others did not changed in ripe mango. Meanwhile, three $\alpha$-amylases were found (comp46096, comp40871, comp40434) highly expressed and did not change during ripening. In mango fruit, it has been shown that these genes have a very important role in fruit quality. Lima et al. (2001) reported that in fruit that showed lower activities of amylase, a spongy tissue was observed, which is a physiological disorder that affects the fruit quality and therefore, the potential for marketing.

\section{Genes related to flavor quality}

Fruit flavor is often dependent on aroma generated by volatile compounds and taste is associated with the sugar/acid ratio. Sucrose, glucose and fructose are the most abundant fruit sugars (Chaimanee and Suntornwat, 1994). In fruit ripening, accumulation of sucrose is due to the activity of the enzyme sucrose phosphate synthase (SPS) and sucrose phosphate phosphatase (SPP). In this study, six SPS genes were identified and they remained constantly expressed from mature-green to ripe mango. Two SPP genes were obtained and one of them (comp47111) was highly expressed in both stages. While seven sucrose synthase (SuSy) genes were identified, one of them (comp45134) was upregulated and the rest did not changed in ripe mango. Recently, Islam et al. (2014) reported the presence of at least six SuSy genes in Citrus, four of them were expressed in fruit juice sacs and mature leaves and two expressed in young leaves. They demonstrated that the Citrus SuSy genes have different spatio-temporal expression patterns.

Mango fruit has more than 300 volatile compounds identified and terpene hydrocarbons (monoterpenes and sesquiterpenes) are the dominant volatile components (Pino et al., 2005). This transcriptome data set contained a total of 29 terpenoid backbone biosynthesis genes, and seven were DE (Table 2). Farnesyl pyrophosphate synthase (FPPS) catalyzes the synthesis of farnesyl pyrophosphate, a key intermediate in sterol and sesquiterpene biosynthesis. Recently, a FPPS gene was identified in mango "Alphonso" and it was expressed during fruit ripening (Kulkarni et al., 2013). An FPPS (comp48152) transcript was identified and showed a 2 -fold increase in expression in ripe mango. Another 
important gene for aroma is the geranylgeranyl pyrophosphate synthase (GGPS), in this study two GGPS genes were identified, one of them (comp40639) was highly expressed in both stages and the other (comp48107) was DE (8-fold change) during ripening. In orange, mono and sesquiterpenes are the major components for flavor, while in climacteric varieties of melon, volatile esters are the predominant components together with sesquiterpenes, norisoprenoids, alcohols, and aldehydes (Sharon-Asa et al., 2003; El Hadi et al., 2013).

During ripening the membranes and the cell wall become permeable allowing the lipoxygenase (LOX) pathway to be active by sequential enzyme steps involving lipoxygenase and hydroperoxide lyase (HPL). Substrates for lipoxygenase, such as linoleic and linolenic acids, are common constituents of plant membranes (Riley etal., 1996). For mangos "Kensington Pride," caprylic, capric, stearic, oleic, linoleic and linolenic acids were found in the pulp of ripe fruit (Lalel et al., 2003). Six LOX genes were found DE in this transcriptome in ripe mango; four genes (comp20603, comp47763, comp40188, comp44212) were upregulated with $6,5,3$, and 3 -fold change, respectively. In tomato, at least five LOX genes have been reported, but there is only one (TomloxC) associated with volatile synthesis and it is expressed during tomato ripening (Heitz etal., 1997; Chen et al., 2004). This TomloxC is $47 \%$ identical with a LOX gene (comp44212) of mango.

\section{Genes associated to color}

Mesocarp in mango fruit changes from green to yellow-orange color due to the accumulation of carotenoids (Tanaka et al., 2008). It has been reported that all-trans- $\beta$-carotene, all-transviolaxanthin and 9-cis-violaxanthin (as dibutyrates) are the principal "Ataulfo" mango carotenoids. During fruit ripening, carotenoids accumulate in an exponential manner, especially all-trans- $\beta$-carotene (Ornelas-Paz et al., 2008). In tomato, the genes encoding enzymes of the carotenoid pathway are regulated both transcriptionally (Klee and Giovannoni, 2011) and post- transcriptionally (Cazzonelli and Pogson, 2010). In this study, 10 genes that encode enzymes of carotenoid metabolism in mango mesocarp were DE; a PSY (comp42694) that carry out the first committed step, was highly expressed in mango and did not changed during ripening. While two identified phytoene desaturases (PDSs; comp46184 and comp44482) and one zeta-carotene desaturase (ZDS; comp39926) did not changed during ripening. However, a lycopene isomerase (MiCRTISO; comp42184) gene presented 4 -fold change in ripe mango; similarly, a tomato SlCRTISO was also up-regulated at the red-ripe stage of fruit development (Smita et al., 2013).

In this study, a lycopene-beta-cyclase ( $L C Y B$; comp42574) transcript presented a -2 fold change in ripe mango. $L C Y B$ is $83 \%$ identical to persimmon fruit $D k L C Y B$ and relative expression levels of $D k L C Y B$ followed a similar expression pattern of mango $L C Y B$. The levels of $D k L C Y B$ decreased during ripening and correlated with the increased contents of $\beta$-cryptoxanthin and $\beta$-carotene in this fruit (Zhao et al., 2011). Two carotenoid $\beta$-hydroxylase (CHYB1 and CHYB2) genes were identified in mango. CHYB1 (comp45152) presented a 4-fold increase in ripe mango; while CHYB2 did not changed during ripening. Zeaxanthin epoxidase (ZEP; comp41313) expression level was down-regulated in ripe mango with -2 fold change. Two additional genes from the isoprenoid pathway that may be associated with color development were identified in this transcriptome, unigene farnesyl diphosphate synthase (comp 48152) and GGPS (comp48107) were differentially expressed with 2 and 8 -fold change, respectively.

\section{qRT-PCR VALIDATION}

In order to validate the DE data obtained by RNA-seq, the following single unigenes were selected to experimentally determine their expression levels by qRT-PCR: 1-aminocyclopropane1-carboxylate oxidase (ACO) comp59876, ethylene receptor 1 (ETR1) comp46286, ethylene response sensor 1 (ERS1) comp32379, ethylene insensitive 4 (EIN4) comp34039, PME comp45079, PL comp63384, expansin (EXP) comp51667,
A

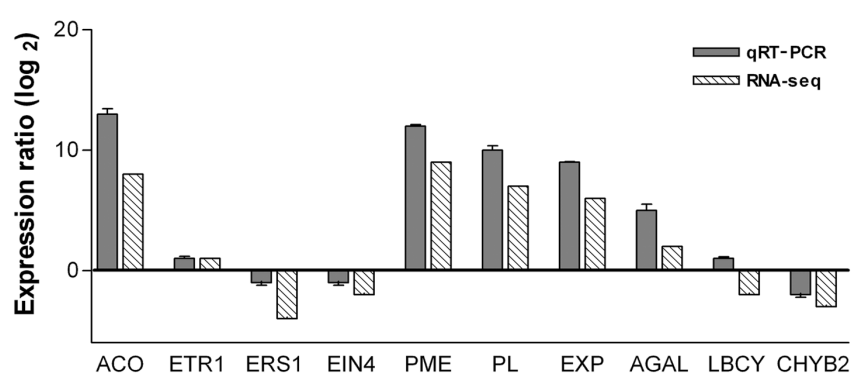

B

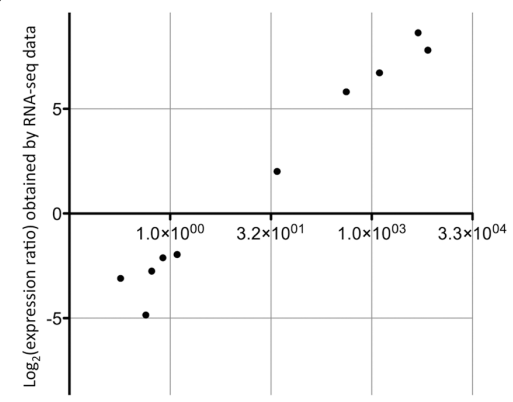

$\log _{2}$ (expression ratio) obtained by qRT-PCR
FIGURE 4 | (A) Expression ratio ( $\log _{2}$ ) obtained by qRT-PCR and RNA-seq of 10 selected genes associated to fruit ripening. ACO, 1-aminocyclopropane-1-carboxylate oxidase (comp59876); ETR1, ethylene receptor (comp46286); ERS1, ethylene receptor sensor (comp32379); EIN4, ethylene insensitive 4 (comp34039); PME, pectin methyl esterase (comp45079); $\mathrm{PL}$, pectate lyase (comp63384); EXP, expansin (comp51667); AGAL, alpha-galactosidase (comp46653); LCBY, Iycopene beta-cyclase (comp42574); and CHYB2, carotenoid beta-hydroxylase 2 (comp39312). GAPDH was used as a reference gene for normalization of qRT-PCR data. Bars represent the error standard $(n=3)$. (B) Correlation between the gene expression ratios obtained from RNA-seq data and qRT-PCR. The RNA-seq $\log _{2}$ value of the expression ratio is shown in the $y$-axis and the qRT-PCR $\log _{2}$ value of the expression ratio in the x-axis. 
alpha-galactosidase (AGAL) comp46653, lycopene beta-cyclase (LCYB) comp42574 and carotenoid beta-hydroxylase 2 (CHYB2) comp39312. The results for gene expression ratios between ripe and mature-green mango are shown in Figure 4A. A linear regression analysis showed an overall correlation coefficient of $R=0.97$, which indicates a high correlation between transcript abundance assayed by $\mathrm{qRT}$-PCR and the transcription profile revealed by RNA-seq data (Figure 4B). The results showed that although the exact fold changes of selected genes at the two data points (maturegreen and ripe mango) varied between RNA-seq and qRT-PCR analyses, trends of gene expression by the two different approaches were consistent. The RNA-seq data reflects what is happening in the cell at the transcriptional level during mango ripening.

\section{CONCLUSION}

The transcriptome of mango mesocarp captured most of the gene space in mango. We were able to identify many differentially expressed genes involved in ripening in mango. The expression data obtained by RNA-seq was favorably validated with the data obtained by qRT-PCR. Unigenes that code for gene products from pathways such as plant hormone signal transduction, starch and sucrose metabolism, galactose metabolism, terpenoid backbone biosynthesis and carotenoid biosynthesis, that allow characteristic changes during fruit ripening were identified in this transcriptome. Such genes can now be used to address particular questions in fruit ripening and allow expansion of the time course to earlier stages of fruit development.

\section{AUTHOR CONTRIBUTIONS}

Conceived and designed the experiments: MAIO, SCF, MDC. Performed the experiments: MDC, ASF, CACV. Analyzed the data: MDC, ASF, AOL, CACV, MAPS, DNK. Contributed reagents/materials/analysis tools: MAIO, ASF, AOL, SCF. Wrote the paper: MAIO, MDC, MAPS, CACV, AOL, DNK.

\section{ACKNOWLEDGMENTS}

MDC and MAPS were supported by a PhD scholarship from CONACyT. Dr. MAIO acknowledges grant CB2012-01-178296 from CONACyT. We thank CIAD personnel: Gerardo Reyna, Felipe Isaac, Luis Leyva, Adalberto Murrieta, José Luis Aguilar, and Martin Peralta for bibliographical and computational support; Monica Villegas, Emmanuel Aispuro, and Francisco Soto for laboratory technical support. We thank Zyanya Lucía Zatarain Barrón and Ma. Fernanda Cornejo Granados for the statistical correlation analysis. We thank the services provided by Unidad Universitaria de Secuenciación Masiva de DNA from IBT-UNAM. We thank Arnulfo Ochoa for computational support and Ing. Ernesto Sanchez from INIFAP for access to mango samples and to Veronica Jimenez for uploading data. We also thank Dr. Frans Tax (University of Arizona) and Dr. Rogerio Sotelo-Mundo (CIAD) for critical reading of the manuscript.

\section{SUPPLEMENTARY MATERIAL}

The Supplementary Material for this article can be found online at: http://www.frontiersin.org/journal/10.3389/fpls.2015.00062/ abstract

\section{REFERENCES}

Ali, Z. M., Armugam, S., and Lazan, H. (1995). $\beta$-Galactosidase and its significance in ripening mango fruit. Phytochemistry 38, 1109-1114. doi: 10.1016/00319422(94)00804-3

Azim, M., Kamran, K., Ishtaiq, A., and Zhang, Y. (2014). Characterization of mango (Mangifera indica L.) transcriptome and chloroplast genome. Plant Mol. Biol. 85, 193-208. doi: 10.1007/s11103-014-0179-8

Bleecker, A. B. (1998). The ethylene-receptor family from Arabidopsis: structure and function. Philos. Trans. R. Soc. Lond. B Biol. Sci. 353, 1405-1412. doi: 10.1098/rstb.1998.0295

Bleecker, A. B. (1999). Ethylene perception and signaling: an evolutionary perspective. Trends Plant Sci. 4, 269-274. doi: 10.1016/S1360-1385(99)01427-2

Bleeker, P. M., Spyropoulou, E. A., Diergaarde, P. J., Volpin, H., De Both, M. T. J., Zerbe, P., et al. (2011). RNA-seq discovery, functional characterization, and comparison of sesquiterpene synthases from Solanum lycopersicum and Solanum habrochaites trichomes. Plant Mol. Biol. 77, 323-336. doi: 10.1007/s11103-011-9813-x

Brummell, D. A., and Harpster, M. H. (2001). Cell wall metabolism in fruit softening and quality and its manipulation in transgenic plants. Plant Mol. Biol. 47, 311340. doi: 10.1007/978-94-010-0668-2-18

Cárdenas-Coronel, W. G., Velez-de la Rocha, R., Siller-Cepeda, J. H., Osuna-Enciso, T., Muy-Rangel, M. D., and Sañudo-Barajas, J. A. (2012). Changes in the composition of starch, pectins and hemicelluloses during the ripening stage of mango (Mangifera indica cv. Kent). Rev. Chapingo Serie Hortic. 18, 5-19.

Cazzonelli, C. I., and Pogson, B. J. (2010). Source to sink: regulation of carotenoid biosynthesis in plants. Trends Plant Sci. 15, 266-274. doi: 10.1016/j.tplants.2010.02.003

Chaimanee, P., and Suntornwat, O. (1994). Changes in carbohydrate content during fruit ripening - a new approach of teaching of carbohydrate chemistry in biochemistry course. Biochem. Educ. 22, 101-102. doi: 10.1016/03074412(94)90098-1

Chen, G., Hackett, R., Walker, D., Taylor, A., Lin, Z., and Grierson, D. (2004). Identification of a specific isoform of tomato lipoxygenase (TomloxC) involved in the generation of fatty acid-derived flavor compounds. Plant Physiol. 136, 2641-2651. doi: 10.1104/pp.104.041608

Chourasia, A., Sane, V. A., and Nath, P. (2006). Differential expression of pectate lyase during ethylene-induced postharvest softening of mango (Mangifera indica var. Dashehari). Physiol. Plant. 128, 546-555. doi: 10.1111/j.1399-3054.2006. 00752.x

Conesa, A., and Götz, S. (2008). Blast2GO: a comprehensive suite for functional analysis in plant genomics. Int. J. Plant Genomics. 2008:619832. doi: $10.1155 / 2008 / 619832$

El Hadi, M. A. M., Zhang, F. J., Wu, F. F., Zhou, C. H., and Tao, J. (2013). Advances in fruit aroma volatile research. Molecules 18, 8200-8229. doi: 10.3390/molecules 18078200

Fabi, J. P., Broetto, S. G., de Silva, S. L., Zhong, S., Lajolo, F. M., and do Nascimento, J. R. (2014). Analysis of papaya cell wall-related genes during fruit ripening indicates a central role of polygalacturonases during pulp softening. PLoS ONE 9:e105685. doi: 10.1371/journal.pone.0105685

Feng, C., Chen, M., Xu, C. J., Bai, L., Yin, X. R., Li, X., et al. (2012). Transcriptomic analysis of Chinese bayberry (Myrica rubra) fruit development and ripening using RNA-Seq. BMC Genomics 13:19. doi: 10.1186/1471-2164-13-19

Gapper, N. E., McQuinn, R. P., and Giovannoni, J. J. (2013). Molecular and genetic regulation of fruit ripening. Plant Mol. Biol. 82, 575-591. doi: 10.1007/s1103013-0050-3

Gerardi, C., Blando, F., Santino, A., and Zacheo, G. (2001). Purification and characterisation of a $\beta$-glucosidase abundantly expressed in ripe sweet cherry (Prunus avium L.) fruit. Plant Sci. 160, 795-805. doi: 10.1016/S0168-9452(00) 00423-4

Goulao, L. F., and Oliveira, C. M. (2008). Cell wall modifications during fruit ripening: when a fruit is not the fruit. Trends Food Sci. Technol. 19, 4-25. doi: 10.1016/j.tifs.2007.07002

Grabherr, M. G., Haas, B. J., Yassour, M., Levin, J. Z., Thompson, D. A., Amit, I., et al. (2011). Full-length transcriptome assembly from RNA-Seq data without a reference genome. Nat. Biotechnol. 29, 644-652. doi: 10.1038/ nbt. 1883

Guo, H., and Ecker, J. R. (2003). Plant responses to ethylene gas are mediated by SCFEBF1/EBF2- dependent proteolysis of EIN3 transcription factor. Cell 115, 667-677. doi: 10.1016/S0092-8674(03)00969-3 
Guo, S., Liu, J., Zheng, Y., Huang, M., Zhang, H., Gong, G., et al. (2011). Characterization of transcriptome dynamics during watermelon fruit development: sequencing, assembly, annotation and gene expression profiles. BMC Genomics 12:454. doi: 10.1186/1471-2164-12-454

Heitz, T., Bergey, D. R., and Ryan, C. A. (1997). A gene encoding a chloroplasttargeted lipoxygenase in tomato leaves is transiently induced by wounding, systemin, and methyl jasmonate. Plant Physiol. 114, 1085-1093. doi: 10.1104/pp.114.3.1085

Islam, M. Z., Hu, X.-M., Jin, L.-F., Liu, Y.-Z., and Peng, S.-A. (2014). Genome-wide identification and expression profile analysis of Citrus sucrose synthase genes: investigation of possible roles in the regulation of sugar accumulation. PLoS ONE 9:e113623. doi: 10.1371/journal.pone0113623

Kanehisa, M., and Goto, S. (2000). KEGG: kyoto encyclopedia of genes and genomes. Nucleic Acids Res. 28, 27-30. doi: 10.1093/nar/28.1.27

Klee, H. J., and Giovannoni, J. J. (2011). Genetics and control of tomato fruit ripening and quality attributes. Annu. Rev. Genet. 45, 41-59. doi 10.1146/annurev-genet-110410-132507

Kobe, B., and Kajava, A. V. (2001). The leucine-rich repeat as a protein recognition motif. Curr. Opin. Struct. Biol. 11, 725-732. doi: 10.1016/S0959440X(01)00266-4

Kulkarni, R., Pandit, S., Chidley, H., Nagel, R., Schmidt, A., Gershenzon, J., et al. (2013). Characterization of three novel isoprenyl diphosphate synthases from the terpenoid rich mango fruit. Plant Physiol. Biochem. 71, 121-131. doi: 10.1016/j.plaphy.2013.07.006

Lalel, H. J. D., Singh, Z., and Tan, S. C. (2003). Maturity stage at harvest affects fruit ripening, quality and biosynthesis of aroma volatile compounds in 'Kensington Pride' mango. J. Hortic. Sci. Biotech. 78, 225-233.

Lashbrook, C. C., Tieman, D. M., and Klee, H. J. (1998). Differential regulation of the tomato ETR gene family throughout plant development. Plant J. 15, 243-252. doi: 10.1046/j.1365-313X.1998.00202.x

Lima, L. C. D. O., Chitarra, A. B., and Chitarra, M. I. F. (2001). Changes in amylase activity starch and sugars contents in mango fruits pulp cv. Tommy Atkins with spongy tissue. Braz. Arch. Biol. Technol. 44, 59-62. doi: 10.1590/S1516-89132001000100008

Livak, K. J., and Schmittgen, T. D. (2001). Analysis of relative gene expression data using real-time quantitative PCR and the 2- $\Delta \Delta$ CT Method. Methods 25, 402-408. doi: 10.1006/meth.2001.1262

Lopez-Gomez, R., and Gómez-Lim, M. A. (1992). A method for extracting intact RNA from fruits rich in polysaccharides using ripe mango mesocarp. HortScience 27, 440-442.

Marguerat, S., and Bähler, J. (2010). RNA-seq: from technology to biology. Cell Mol. Life Sci. 67, 569-579. doi: 10.1007/s00018-009-0180-6

Marin-Rodriguez, M. C., Smith, D. L., Manning, K., Orchard, J., and Seymour, G. B. (2003). Pectate lyase gene expression and enzyme activity in ripening banana fruit. Plant Mol. Biol. 51, 851-857. doi: 10.1023/A:1023057202847

Mehar, H. A., and Pravendra, N. (2005). Expression of multiple forms of polygalacturonase gene during ripening in banana fruit. Plant Physiol. Biochem. 43, 177-184. doi: 10.1016/j.plaphy.2005.01.011

McQueen-Mason, S. J., and Cosgrove, D. J. (1995). Expansin mode of action on cell walls. Analysis of wall hydrolysis, stress relaxation, and binding. Plant Physiol. 107, 87-100. doi: 10.1104/pp.107.1.87

Molina-Hidalgo, F. J., Franco, A. R., Villatoro, C., Medina-Puche, L., Mercado, J. A., Hidalgo, M. A., et al. (2013). The strawberry (Fragaria $\times$ ananassa) fruit-specific rhamnogalacturonate lyase 1 (FaRGLyase1) gene encodes an enzyme involved in the degradation of cell-wall middle lamellae. J. Exp. Bot. 64, 1471-1483. doi: $10.1093 /$ jxb/ers386

Muy-Rangel, D., Espinoza-Valenzuela, B., Siller Cepeda, J., Sanudo-Barajas, J. A., Valdez-Torres, B., and Osuna-Enciso, T. (2009). Effect of 1methylcyclopropene (1-MCP) and an edible coating on enzyme activity and postharvest quality of mango 'Ataulfo.' Rev. Fitotecnia Mexicana 32, 53-60.

Nath, P., Trivedi, P. K., Sane, V. A., and Sane, A. P. (2006). "Role of the ethylene in fruit ripening," in Ethylene Action in Plants, ed. N. A. Khan (Berlin: Springer), 151-184. doi: 10.1007/978-3-540-32846-9_8

Oomen, R. J. F. J., Doeswijk-Voragen, C. H. L., Bush, M. S., Vincken, J. P., Borkhardt, B., Van Den Broek, L. A. M., et al. (2002). In muro fragmentation of the rhamnogalacturonan I backbone in potato (Solanum tuberosum L.) results in a reduction and altered location of the galactan and arabinan side-chains and abnormal periderm development. Plant J. 30, 403-413. doi: 10.1046/j.1365-313X.2002.01296.x

Ornelas-Paz, J. J., Yahia, E. M., and Gardea, A. A. (2008). Changes in external and internal color during postharvest ripening of 'Manila'and 'Ataulfo' mango fruit and relationship with carotenoid content determined by liquid chromatographyAPcl+-time-of-flight mass spectrometry. Postharvest Biol. Technol. 50, 145-152. doi: 10.1016/j.postharvbio.2008.05.001

Pino, J. A., Mesa, J., Muñoz, Y., Martí, M. P., and Marbot, R. (2005). Volatile components from mango (Mangifera indica L.) cultivars. J. Agric. Food Chem. 53, 2213-2223. doi: 10.1021/jf0402633

Prasanna, V., Prabha, T. N., and Tharanathan, R. N. (2005). Multiple forms of $\beta$ galactosidase from mango (Mangifera indica L. Alphonso) fruit pulp. J. Sci. Food Agric. 85, 797-803. doi: 10.1002/jsfa.2028

Prasanna, V., Prabha, T. N., and Tharanathan, R. N. (2006). Multiple forms of polygalacturonase from mango (Mangifera indica L. cv. Alphonso) fruit. Food Chem. 95, 30-36. doi: 10.1016/j.foodchem.2004. 12.014

Riley, J., Willemot, C., and Thompson, J. E. (1996). Lipoxygenase and hydroperoxide lyase activities in ripening tomato fruit. Postharvest Biol. Technol. 7, 97-107. doi: 10.1016/0925-5214(95)00032-1

Sane, V. A., Chourasia, A., and Nath, P. (2005). Softening in mango (Mangifera indica cv. Dashehari) is correlated with the expression of an early ethylene responsive, ripening related expansin gene MiExpA1. Postharvest Biol. Technol. 38, 223-230. doi: 10.1016/j.postharvbio.2005.07.008

Schaller, G. E., and Kieber, J. J. (2002). Ethylene. Arabidopsis Book 1:e0071. doi: 10.1199/tab.0071

Sharon-Asa, L., Shalit, M., Frydman, A., Bar, E., Holland, D., Or, E., et al. (2003). Citrus fruit flavor and aroma biosynthesis: isolation, functional characterization, and developmental regulation of Cstps1, a key gene in the production of the sesquiterpene aroma compound valencene. Plant J. 36, 664-674. doi: 10.1046/j.1365-313X.2003.01910.x

Singh, P., and Dwivedi, U. N. (2008). Purification and characterisation of multiple forms of polygalacturonase from mango (Mangifera indica cv. Dashehari) fruit. Food Chem. 111, 345-349. doi: 10.1016/j.foodchem.2008. 03.072

Singh, Z., Singh, R. K., Sane, V. A., and Nath, P. (2013). Mango-postharvest biology and biotechnology. CRC Crit. Rev. Plant Sci. 32, 217-236. doi: 10.1080/07352689.2012.743399

Smita, S., Rajwanshi, R., Lenka, S. K., Katiyar, A., Chinnusamy, V., and Bansal, K. C. (2013). Expression profile of genes coding for carotenoid biosynthetic pathway during ripening and their association with accumulation of lycopene in tomato fruits. J. Genet. 92, 363-368. doi: 10.1007/s12041-0130275-6

Stirnimann, C. U., Petsalaki, E., Russell, R. B., and Müller, C. W. (2010). WD40 proteins propel cellular networks. Trends Biochem. Sci. 35, 565-574. doi: 10.1016/j.tibs.2010.04.003

Tanaka, Y., Sasaki, N., and Ohmiya, A. (2008). Biosynthesis of plant pigments: anthocyanins, betalains, and carotenoids. Plant J. 54, 733-749. doi: 10.1111/j.1365-313X.2008.03447.x

Tatusov, R. L., Galperin, M. Y., Natale, D. A., and Koonin, E. V. (2000). The COG database: a tool for genome-scale analysis of protein functions and evolution. Nucleic Acids Res. 28, 33-36. doi: 10.1093/nar/28.1.33

Tieman, D. M., and Klee, H. J. (1999). Differential expression of two novel members of the tomato ethylene-receptor family. Plant Physiol. 120, 165-172. doi: 10.1104/pp.120.1.165

UniProt Consortium. (2014). Activities at the Universal Protein Resource (UniProt). Nucleic Acids Res. 42, D191-D198. doi: 10.1093/nar/gkt1140

White, P. J. (2002). Recent advances in fruit development and ripening: an overview. J. Exp. Bot. 53, 1995-2000. doi: 10.1093/jxb/erf105

Wu, H. X., Jia, H. M., Ma, X. W., Wang, S. B., Yao, Q. S., Xu, W. T., et al. (2014a). Transcriptome and proteomic analysis of mango (Mangifera indica Linn) fruits. J. Proteomics 105, 19-30. doi: 10.1016/j.jprot.2014.03. 030

Wu, J., Xu, Z., Zhang, Y., Chai, L., Yi, H., and Deng, X. (2014b). An integrative analysis of the transcriptome and proteome of the pulp of a spontaneous late-ripening sweet orange mutant and its wild type improves our understanding of fruit ripening in Citrus. J. Exp. Bot. 65, 1651-1671. doi: 10.1093/jxb/e ru044 
Yashoda, H. M., Prabha, T. N., and Tharanathan, R. N. (2006). Mango ripening: changes in cell wall constituents in relation to textural softening. J. Sci. Food Agric. 86, 713-721. doi: 10.1002/jsfa.2404

Yu, K., Xu, Q., Da, X., Guo, F., Ding, Y., and Deng, X. (2012). Transcriptome changes during fruit development and ripening of sweet orange (Citrus sinensis). BMC Genomics 13:10. doi: 10.1186/1471-2164-13-10

Zhao, D., Zhou, C., and Tao, J. (2011). Carotenoid accumulation and carotenogenic genes expression during two types of persimmon fruit (Diospyros kaki L.) development. Plant Mol. Biol. Rep. 29, 646-654. doi: 10.1007/s11105-010-0272-3

Zheng, X., Jing, G., Liu, Y., Jiang, T., Jiang, Y., and Jianrong, L. I. (2012). Expression of expansin gene, $M i \operatorname{ExpA}$, and activity of galactosidase and polygalacturonase in mango fruit as affected by oxalic acid during storage at room temperature. Food Chem. 132, 849-854. doi: 10.1016/j.foodchem.2011.11.049

Conflict of Interest Statement: The authors declare that the research was conducted in the absence of any commercial or financial relationships that could be construed as a potential conflict of interest.
Received: 19 November 2014; accepted: 24 January 2015; published online: 18 February 2015.

Citation: Dautt-Castro M, Ochoa-Leyva A, Contreras-Vergara CA, Pacheco-Sanchez MA, Casas-Flores S, Sanchez-Flores A, Kuhn DN and Islas-Osuna MA (2015) Mango (Mangifera indica L.) cv. Kent fruit mesocarp de novo transcriptome assembly identifies gene families important for ripening. Front. Plant Sci. 6:62. doi: 10.3389/fpls.2015.00062

This article was submitted to Plant Genetics and Genomics, a section of the journal Frontiers in Plant Science.

Copyright (c) 2015 Dautt-Castro, Ochoa-Leyva, Contreras-Vergara, Pacheco-Sanchez, Casas-Flores, Sanchez-Flores, Kuhn and Islas-Osuna. This is an open-access article distributed under the terms of the Creative Commons Attribution License (CC BY). The use, distribution or reproduction in other forums is permitted, provided the original author(s) or licensor are credited and that the original publication in this journal is cited, in accordance with accepted academic practice. No use, distribution or reproduction is permitted which does not comply with these terms. 